\title{
Clopidogrel-Induced Gastric Injury in Rats is Attenuated by Stable Gastric Pentadecapeptide BPC I57
}

This article was published in the following Dove Press journal:

Drug Design, Development and Therapy

\author{
Hailu Wu (iD) ${ }^{1,2}$ \\ Ming $\mathrm{Wei}^{2}$ \\ Nan $\mathrm{Li}^{2}$ \\ Qin $\mathrm{Lu}^{2}$ \\ Sachin Mulmi Shrestha' \\ Jiacheng $\operatorname{Tan}^{2}$ \\ Zhenyu Zhang ${ }^{3}$ \\ Guoqiu Wu ${ }^{4,5}$ \\ Ruihua $\mathrm{Shi}^{2}$ \\ 'Medical School of Southeast University, \\ Nanjing 210009, People's Republic of \\ China; ${ }^{2}$ Department of Gastroenterology, \\ Zhongda Hospital Affiliated to Southeast \\ University, Nanjing 210009, People's \\ Republic of China; ${ }^{3}$ Division of \\ Gastroenterology, Department of \\ Medicine, Nanjing Medical University \\ Nanjing First Hospital, Nanjing 210009 , \\ People's Republic of China; ${ }^{4}$ Center of \\ Clinical Laboratory Medicine, Zhongda \\ Hospital, Southeast University, Nanjing \\ 210009, People's Republic of China; \\ 5 Jiangsu Provincial Key Laboratory of \\ Critical Care Medicine, Nanjing 210009, \\ People's Republic of China
}

Correspondence: Ruihua Shi

Department of Gastroenterology,

Zhongda Hospital Affiliated to Southeast

University, Nanjing 210009 , People's

Republic of China

Tel +862583262833

Fax +862583262830

Email ruihuashi@I26.com

Guoqiu Wu

Center of Clinical Laboratory Medicine,

Zhongda Hospital, Southeast University,

Nanjing 210009, People's Republic of

China

Tel +86-2583272150

Email nationball@I63.com
Aim: Although Clopidogrel is safe in healthy volunteers, it can induce recurrence of gastric ulcers in high-risk patients. Here, we investigated the protective effect of the natural product, stable gastric pentadecapeptide 157 (BPC 157) on Clopidogrel-induced gastric injury.

Methods: We used acetic acid to induce gastric ulcer in Sprague Dawley rats. Clopidogrel alone or in combination with BPC 157 or L-NAME (nitric oxide system blockade) were administered after healing of acetic acid-induced ulcer. One percent methylcellulose solution was used as control. Ulcer recurrence rate and the ulcer index were compared between these groups. Gastric mucosal apoptosis rate, microscopic inflammation activity and angiogenesis markers vascular endothelial growth factor $\mathrm{A}(V E G F-A)$ and $C D 34$ were examined by TUNEL, histological evaluations (HE) and immunohistochemistry (IHC). Pathways involved, expressions of endoplasmic reticulum (ER) stress apoptosis marker $C H O P$, angiogenic markers $V E G F-A$ and its receptor $V E G F R 1$, and endothelial NO synthase (eNOS) were all analyzed by Western blot.

Results: This study indicated that Clopidogrel significantly induced the gastric ulcers recurrence, severe inflammation and ER stress related apoptosis of the gastric mucosa, suppressed the synthesis of angiogenic markers and eNOS. Furthermore, Clopidrogel intervention resulted in the activation of protein kinase $\mathrm{B}(A K T)$ and $\mathrm{p} 38$ mitogen-activated protein kinase ( $p 38 / M A P K)$. BPC 157 attenuated the gastric mucosal damage caused by Clopidogrel and reversed these molecular effects. However, NO blockade L-NAME weakened the protective effect and thus the molecular effects of BPC 157 on gastric mucosa.

Conclusion: In conclusion, these results suggest that BPC 157 inhibited Clopidogrelinduced gastric mucosa injury partially by inhibition of gastric mucosa cell ER stressmediated apoptosis and inflammation, and promoting gastric mucosa angiogenesis via $V E G F-A / V E G F R 1$ mediated- $A K T / p 38 / M A P K$ signaling pathways.

Keywords: clopidogrel, BPC 157, angiogenesis, AKT, p38/MAPK

\section{Introduction}

Clopidogrel is an oral irreversible $P 2 Y 12$ receptor antagonist which inhibits platelet aggregation and is widely used by patients with acute coronary syndromes or after percutaneous coronary intervention. ${ }^{1,2}$ Although Clopidogrel cannot cause damage to the gastric mucosa in healthy volunteers, ${ }^{3}$ it can increase the bleeding rate in patients at high risk of ulcers ${ }^{4,5}$ and induce recurrence of a healed ulcer during a 6-month follow-up period. ${ }^{6}$ The mechanism of Clopidogrel on gastric mucosa injury is still unclear. Previous studies have confirmed that Ticlopidine, another P2Y12 receptor 
antagonist, delays the healing of rats' ulcers by inhibiting ADP-induced platelets aggregation. ${ }^{7,8}$ In our previous studies, we found that Clopidogrel inhibits human gastric mucosal epithelial cell line GES-1 proliferation then further disrupt the tight junction structure (TJs) of gastric epithelium, ${ }^{9}$ through activation of endoplasmic reticulum (ER) stress-induced apoptosis via $M K P-5$-mediated $p 38 /$ MAPK phosphorylation. ${ }^{10,11}$

It is well known that the reconstruction of epithelial cells and submucosal connective tissues is the pathophysiological basis of gastric ulcer healing, involving cell proliferation and angiogenesis. ${ }^{12,13}$ Angiogenesis stimulates wound healing by forming new blood vessels from pre-existing vessels, within a few days, these are organized into a microvascular network throughout the granulation tissue. Numerous papers have reported that stable gastric pentadecapeptide BPC 157 can regulate angiogenesis during the healing process by rescuing the duodenal lesions in rats through interacting with the nitric oxide (NO) system and reducing of free radical formation, ${ }^{14}$ and also can resolve pringle maneuver ${ }^{15}$ and hippocampal ischemic damage in rats due to ischemia or reperfusion. ${ }^{16}$ Its strong angiogenic effect is mainly via activation of the $V E G F$ AKT-eNOS signaling pathway. ${ }^{17}$ Furthermore, BPC 157 has also been demonstrated to promote vascular endothelial and tendon cell proliferation and migration. ${ }^{18,19}$

As an anti-ulcer peptidergic agent, BPC 157 is also effective both in the upper and lower gastrointestinal tract and now proven to be safe in inflammatory bowel disease (IBD) in clinical Phase II trials, with no toxicity reported. ${ }^{20,21}$ For nonsteroidal anti-inflammatory drugs $\left(\right.$ NSAID $\left._{\mathrm{S}}\right)$ cytotoxicity such as gastrointestinal, liver and brain lesions, BPC 157 counteraction includes both $C O X-1$ and $C O X-2$ blockers. ${ }^{22-26}$ It can even counteract prolongation of bleeding and thrombocytopenia caused by aspirin. $^{27,28}$ BPC157 can repair mucosal integrity and endothelial damage better than sucralfate. ${ }^{29}$ Besides that, BPC 157 also has a stronger effect on promoting angiogenesis and granulation tissue regeneration than $\mathrm{H} 2-$ blockers, omeprazole or even sucralfate. ${ }^{30}$

Sprague Dawley rats are the most widely used animal model for human gastrointestinal disorder research, and the physiology of their stomach has been thoroughly studied and is well known. This study aimed to investigate whether BPC 157 has a protective effect on gastric mucosal damage caused by Clopidogrel in Sprague Dawley rats and further elucidate its molecular mechanism.

\section{Materials and Methods}

\section{Animals}

Adult male Sprague Dawley rats (12-weeks old, $300 \pm 10 \mathrm{~g}$ ) were purchased from Animal Experimental Center, Zhejiang, China. The rats were fed in the standard SPF environment, housed at a temperature of $23^{\circ} \mathrm{C}$ and in a $12 \mathrm{~h}$ light-dark cycle. All rats were given standard food and tap water. The Committee for the Use of Live Animals in Southeast University approved the use of animals in this study (No: 20,181,130,007). All animal housing and handling were conducted in compliance with the guidelines of Southeast University and the National Institutes of Health guide for the care and use of Laboratory animals (NIH Publications No. 8023, revised 1978).

\section{Drugs, Chemicals and Reagent Kits}

Clopidogrel was purchased from MedChen Express (New Jersey, MCE, USA) and suspended in $1 \%$ methylcellulose. Synthesized BPC 157 and L-NAME were purchased from Modong Gene (Nanjing, Jiangsu, China) and SigmaAldrich (Sigma-Aldrich Biotechnology, St. Louis, MO, USA) respectively, both dissolved in saline. Most of the other chemicals and related drugs were obtained from Sigma-Aldrich unless needed to be declared otherwise.

\section{Induction of Rats' Gastric Ulcers by Acetic Acid}

The Sprague Dawley rats' gastric ulcers were induced by acetic acid by following the method of S. Okabe ${ }^{31}$ with slight modifications. After the rats were anesthetized with pentobarbital (80 mg/kg i.p.), a laparotomy along the midline was performed and the stomach was fully exposed. Then, one end of the cylindrical plastic tube $(10 \mathrm{~mm}$ diameter) was adhered to the stomach serosal part, and $80 \%$ acetic acid was injected at the other end for 1 minute. After surgery, the rats were fasted for $12 \mathrm{~h}$ to reduce complications such as abdominal distension and perforation after eating. Then eight rats were sacrificed in every 5 days, and the size of ulcers was measured until the ulcers were completely healed (Figure S1).

\section{Experimental Design}

25 days after gastric ulcer induction, all ulcers were determined completely healed then the other rats were randomly divided into four groups each containing 10 rats. Clopidogrel $10 \mathrm{mg} / \mathrm{kg}$ intragastric (i.g.) once daily, BPC $157 \quad 10 \mathrm{ng} / \mathrm{kg}$ intraperitoneally (i.p.) once daily and L-NAME $5 \mathrm{mg} / \mathrm{kg}$ intraperitoneally (i.p.) once daily were 
given individually or combined at the same time when the rats were under starvation at 8:00am. The administration details are as follows: control group (1\% methylcellulose solution i.g. once daily); Clopidogrel group (Clopidogrel $10 \mathrm{mg} / \mathrm{kg}$ i.g. once daily) ${ }^{8}$ Clopidogrel + BPC 157 group (Clopidogrel $10 \mathrm{mg} / \mathrm{kg}$ i.g. combined with BPC $15710 \mathrm{ng} /$ kg i.p. ${ }^{25}$ once daily); Clopidogrel + BPC $157+$ L-NAME group (Clopidogrel $10 \mathrm{mg} / \mathrm{kg}$ i.g., BPC $15710 \mathrm{ng} / \mathrm{kg}$ i.p. and L-NAME $5 \mathrm{mg} / \mathrm{kg}$ i.p. ${ }^{25}$ once daily). All rats were sacrificed by cervical dislocation after 30 days of drug administration. The gastric ulcer indexes were calculated according to the method described by Antonisamy et al $^{32}$ (Figure S2).

\section{Histological Evaluations (HE) and Immunohistochemistry (IHC)}

Gastric tissue samples fixed in paraformaldehyde were embedded in paraffin, then dyed with hematoxylin and eosin (H\&E). Histological evaluation was performed under an Olympus BH-2 photomicroscope. Gastric injury was graded using a scoring system as previously described. ${ }^{33}$ It was scored using a scale ranging from 0 to 3 (0: none, 1: mild, 2: moderate, and 3: severe) for each criterion. The total score was 12. In addition, IHC for $V E G F-A$ (Proteintech, Chicago, IL, USA) and CD34 (Proteintech, Chicago, IL, USA) were performed using the automated IHC system (Hoffman La-Roche Ltd., Basel, Switzerland). All scores were assessed using the semiquantitative $\mathrm{H}$ scoring approach by two experienced pathologists who were blinded for the assessment.

\section{TUNEL Assay}

Apoptosis of rats' gastric epithelial cells was evaluated using TUNEL apoptosis assay kit (Vazyme, Nanjing, Jiangsu, China) according to the manufacturers' instructions. The apoptotic cells showed intense dark nuclear staining. The proportion of the dark nuclear staining cells was evaluated by counting the cells stained with TUNEL divided by the total number of cells. One thousand nuclei were evaluated. Three independent experiments were performed blindly by two experienced pathologists.

\section{Western Blotting}

The gastric mucosal tissues of rats were lysed in lysis buffer. Total proteins of each sample were separated on a 10\% SDS-PAGE gel and electrophoretically transferred onto a PVDF membrane (EMD Millipore, Billerica, MA, USA). Then the membranes were blocked with 5\% skim milk for 2 hours. This step was followed by incubation with primary antibody overnight at $4^{\circ} \mathrm{C}$. The primary antibodies used were anti-VEGF-A (Santa Cruz Biotechnology, Santa Cruz, CA, USA), anti-VEGFR1 (Cell Signaling Technology, Berkeley, CA, USA), antipVEGFR1 (Cell Signaling Technology, Berkeley, CA, USA), anti-AKT (Santa Cruz Biotechnology, Santa Cruz, CA, USA), anti-pAKT (Santa Cruz Biotechnology, Santa Cruz, CA, USA), anti-ERK (Cell Signaling Technology, Berkeley, CA, USA), anti-pERK (Cell SignalingT echnology, Berkeley, CA, USA), anti-p38 (Cell Signaling Technology, Berkeley, CA, USA), anti-p-p38 (Cell Signaling Technology, Berkeley, CA, USA), anti-eNOS (Proteintech, Chicago, IL, USA), anti-p-eNOS (Proteintech, Chicago, IL, USA), anti-CHOP (Cell Signaling Technology, Berkeley, CA, USA) and anti- $\beta$ actin (Cell Signaling Technology, Berkeley, CA, USA). Then the membranes were incubated with secondary antibody at room temperature for 2 hours. The enhanced chemiluminescence (ECL) kit was used for visualization. The results were semi-quantified by the Gel and Graph Digitizing System (Silk Scientific, Orem, UT, USA). All experiments were repeated for three times.

\section{Statistical Analysis}

All statistical analyses were performed using SPSS 19.0 (IBM Corporation, Armonk, NY, USA) software. All data were presented as mean $\pm \mathrm{SD}$. The independent samples $t$-test was used in analyzing the difference of means between two groups. One-way analysis of variance was used for comparison among three or more groups. Statistical significance was determined at $p$ value $<0.05$.

\section{Results}

\section{Healing of Acetic Acid-Induced Gastric Ulcers in Rats}

Ng's Study has shown that $12 \%$ of patients who had a history of gastrointestinal bleeding developed bleeding again after taking Clopidogrel for 1 year, and the lesions during re-bleeding were the same as previous. ${ }^{34}$ It suggest that Clopidogrel causes re-bleeding only in patients with underlying mucosal injury or scarring. ${ }^{6,35}$ In order to comply with the clinical facts, in this study, we firstly used acetic acid to induce gastric ulcers, then Clopidogrel was introduced after the ulcers were completely healed. We found that after 25 days, the rat gastric mucosa healing rate reached up to $100 \%$ (Table 1). The 
Table I Gastric Mucosal Healing After Gastric Ulcer Induced by Acetic Acid in Rats

\begin{tabular}{|l|l|l|}
\hline Days & Healing Ratio & Ulcer Size $\left(\mathbf{m m}^{\mathbf{2}}\right)$ \\
\hline 5 & $0 / 8$ & $24.48 \pm 1.02$ \\
10 & $0 / 8$ & $18.48 \pm 1.06^{* * *}$ \\
15 & $5 / 8$ & $7.62 \pm 1.00^{\# \#}$ \\
20 & $7 / 8$ & $3.32 \pm 0.63^{\Delta \Delta \Delta}$ \\
25 & $8 / 8$ & $0.00 \pm 0.00^{+++}$ \\
\hline
\end{tabular}

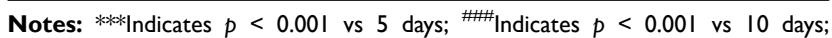
${ }^{\Delta \Delta \Delta}$ Indicates $p<0.001$ vs 15 days; ${ }^{+++}$Indicates $p<0.001$ vs 20 days.

average ulcer size became smaller gradually until healed completely after 25 days $(p<0.001)$.

\section{BPC I57 Protects Gastric Mucosa from Clopidogrel-Induced Ulcer Recurrence}

After healing of acetic acid-induced gastric ulcer in 25 days, Clopidogrel was re-used to induce recurrence of the gastric ulcer. Then the effect of BPC 157 on Clopidogrel-induced gastric mucosal damage was assessed. The results showed no visible lesions in control group. Compared to the control group, oral administration of Clopidogrel for 30 days caused gastric ulcer in $60 \%$ of rats, serious mucosal erosions and erythemas. However, Clopidogrel co-treatment with BPC 157 significantly reduced the gastric ulcer recurrence rate and ulcer index, and the formations of mucosal erosions and erythemas were also rare. Interestingly, the nitric oxide (NO) system blockade L-NAME can partially reverse this protective effect including reduction of gastric ulcer recurrence rate and ulcer indexes induced by BPC 157 (Table 2, Figure 1). These results clearly demonstrated the protective effects of BPC 157 in Clopidogrel-induced gastric mucosal damage, and the NO system blockade L-NAME could partially attenuate these protective effects of BPC 157 on gastric mucosa. It suggests that the NO system may be involved

Table 2 The Protective Effect of BPC 157 on ClopidogrelInduced Gastric Ulcer Recurrence and Mucosal Damage

\begin{tabular}{|l|l|l|}
\hline Group & Ulcer Recurrence Ratio & Ulcer Index \\
\hline Control & $0 / 10$ & $0.00 \pm 0.00$ \\
Clopidogrel & $6 / 10$ & $11.83 \pm 1.17^{* *}$ \\
Clopidogrel + BPC & $2 / 10$ & $4.50 \pm 0.7 I^{\#}$ \\
I57 & & \\
Clopidogrel + BPC & $5 / 10$ & $10.20 \pm 0.84^{\Delta}$ \\
I57 + L-NAME & & \\
\hline
\end{tabular}

Notes: **Indicates $p<0.01$ vs Control group; ${ }^{\#}$ Indicates $p<0.05$ vs Clopidogrel group; ${ }^{\Delta}$ Indicates $p<0.05$ vs Clopidogrel + BPC 157 group. in the protective effect of BPC 157 on Clopidogrelinduced gastric mucosal injury.

\section{BPC 157 Attenuates Clopidogrel-Induced Gastric Mucosa Cells Inflammation}

As presented in Figure 2, the control group had normal architecture and integrity of gastric mucosa and no infiltration of the inflammatory cells in gastric glands (Figure 2A). In contrast, Clopidogrel treatment markedly induced gastric mucosal erosions, featuring severe hyperemia, epithelial cell loss, disturbed glandular structure, damaged crypts, irregular arrangement and inflammatory cells infiltration (Figure 2A). These histological changes were partially reversed by BPC 157 mainly manifested in the reduction in inflammatory cell infiltration, hyperemia and epithelial cell loss (Figure 2A). Not surprisingly, L-NAME could partially weaken the protective effect of BPC 157 on gastric mucosa (Figure 2A). The results of histological microscopic lesion score are shown in Figure 2B.

\section{BPC 157 Attenuates Clopidogrel-Induced Gastric Mucosa Cells Apoptosis}

In our previous study, we have confirmed that Clopidogrel can stimulate ER stress response then induce gastric mucosal epithelial cell apoptosis, and further disrupt gastric mucosal epithelial barrier. ${ }^{10}$ TUNEL analysis was then used to determine if BPC 157 inhibit gastric mucosa injury via suppression of cell apoptosis. As shown in Figure 3, Clopidogrel induced the apoptosis of gastric mucosal cells compared with the control group (Figure 3A). BPC 157 significantly attenuated the Clopidogrel-induced apoptosis compared with the Clopidogrel-only group (Figure 3A). However, after administration of L-NAME the gastric mucosa apoptotic cells increased again (Figure 3A). The ratio of apoptotic cells in each group is shown in Figure $3 \mathrm{~B}$. This data is consistent with other reports showing that the NO system response acts as an anti-inflammatory agent through inhibition of apoptosis on cells. ${ }^{36}$

\section{BPC 157 Promotes Angiogenesis Through NO Response Following Clopidogrel-Induced Gastric Ulcer}

Our above results confirmed that BPC 157 has a protective effect on Clopidogrel-induced gastric mucosal damage, and the specific mechanism in particular related with the NO response. We all know that NO 

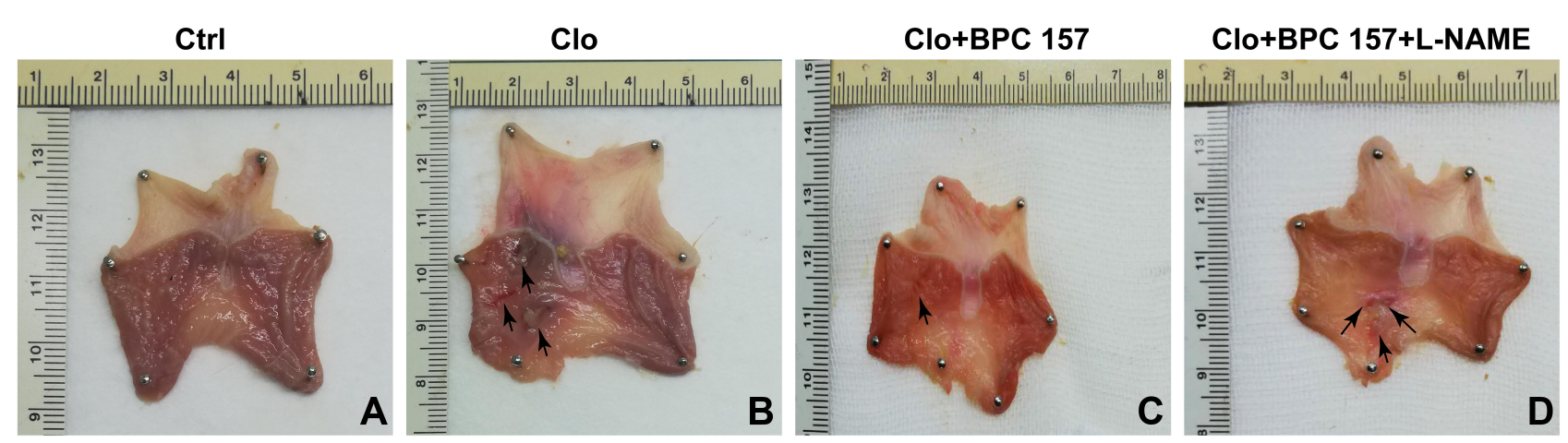

Figure I Representative gross morphologies of stomach at the time of necropsy. (A) Control group (I\% methylcellulose solution) without visible lesions. (B) The Clopidogrel (10 mg/kg) group with severe lesions and deep ulcers. (C) Clopidogrel (10 mg/kg) plus BPC I57 (I0 ng/kg) treatment group with very few lesions and superficial ulcers. (D) L-NAME (5 mg/kg) group with more lesions and ulcers compared with the Clopidogrel (10 mg/kg) plus BPC I57 (I0 ng/kg) treatment group. Arrows: lesions and ulcers.

plays a crucial role in $V E G F-A$-dependent angiogenesis $^{37}$ and the angiogenesis is necessary and most important for ulcer repair. So, next, we performed the immunohistochemical examination of rats' gastric mucosa for the angiogenesis markers VEGF-A and $C D 34$ (Figure 4). In Clopidogrel group, both the levels of $V E G F-A$ and $C D 34$ were decreased compared with control group. In the BPC 157 co-treatment group, the levels of these two angiogenesis markers were all upregulated compared with Clopidogrel-only group. The NO synthase blocker L-NAME inhibited up-regulation of angiogenesis markers VEGF-A and CD34 by BPC 157, suggesting that BPC 157 promotes angiogenesis and then further repair the gastric mucosa through NO response.

\section{The Angiogenesis is Promoted by BPC 157 on Gastric Mucosa via VEGF-A Mediated AKT and P38/MAPK Signaling Pathways}

Considering that $A K T, p 38 / M A P K$ and extracellular regulated protein kinases $(E R K / M A P K)$ are downstream targets of $V E G F-A^{38-40}$ and also the classic pathways that regulate apoptosis and inflammation, we sought to investigate the molecular mechanism by using western-blot analysis. Our results showed that Clopidogrel downregulated the $V E G F-A$ and $V E G F R 1$, subsequently inactivated $A K T$ signaling pathway. However, it was also associated with phosphorylation of $p 38 / M A P K$ and $E R K / M A P K$; furthermore, BPC 157 could significantly
A

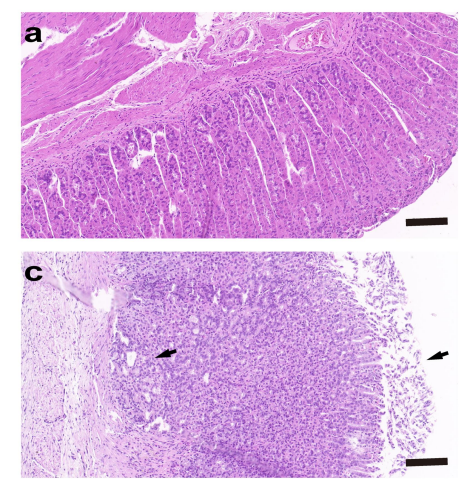

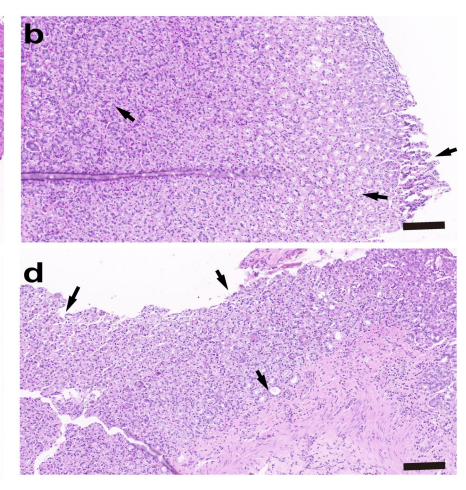

B

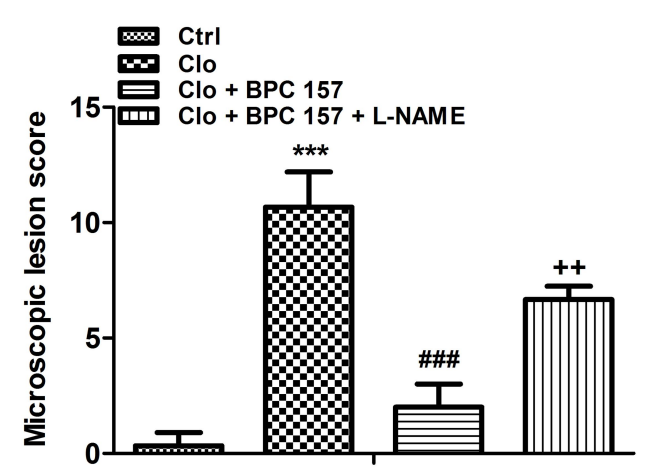

Figure 2 (A) Histopathological features and lesion score of stomach. a, Control group showed intact mucosal lining of flattened epithelial cells. Mucosal crypts appear arranged normally; b, The Clopidogrel group showed denudation of lining epithelium, areas of hyperemia, disturbed glandular structure, damaged crypts, irregular arrangement and inflammatory cells infiltration; c, Clopidogrel plus BPC I57 group with minimal changes compared to Clopidogrel group; d, L-NAME exposed mucosal tissue showed similar pathological changes as in Clopidogrel group. Scale bar $=200 \mu \mathrm{m}$. The lesion score is shown in (B). $* * * P<0.00 \mathrm{I}$ vs Control; ${ }^{*} P<0.00 \mathrm{I}$ vs Control + Clopidogrel; ${ }^{++} P<0.01$ vs Control + Clopidogrel + BPC I57. 
A
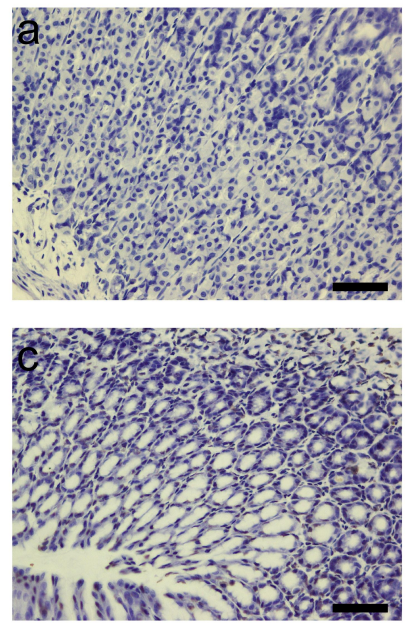
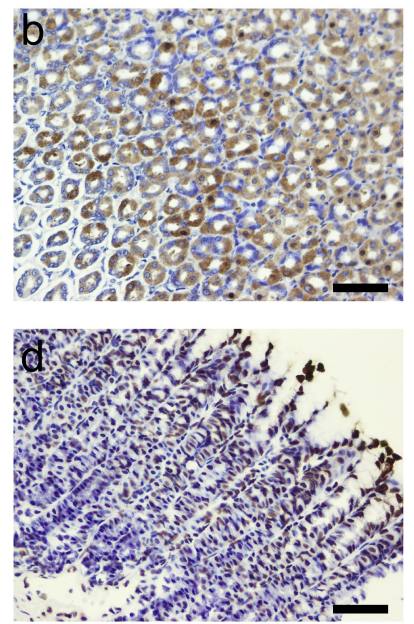

B

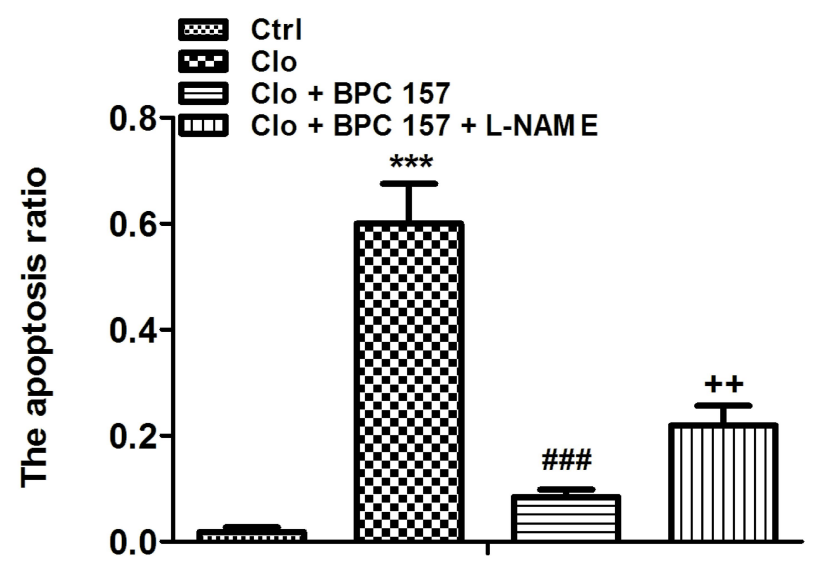

Figure 3 TUNEL staining. Epithelial apoptosis in gastric mucosa was evaluated by TUNEL staining. (A) Representative images of TUNEL staining of stomach from rats in four groups. a, Control group; b, Clopidogrel group; c, Clopidogrel plus BPC I57 group; d, Clopidogrel plus BPC 157 and L-NAME group. Scale bar $=200 \mu \mathrm{m}$. (B) Ratio of TUNEL-positive (brown) cells in each group was shown. $* * * P<0.001$ vs Control; ${ }^{\# \#} P<0.001$ vs Clopidogrel; ${ }^{++} P<0.01$ vs Clopidogrel + BPC I57.

up-regulate the $V E G F-A$ and $V E G F R 1$, paralleled by the phosphorylation of $A K T$, and inactivation of $p 38 / M A P K$ and $E R K / M A P K$ signaling pathways. More importantly, the NO synthase blocker L-NAME can reverse these effects of $A K T$ and $p 38 / M A P K$ signaling pathways induced by BPC 157 , but cause no changes on ERK/ $M A P K$. Therefore, we concluded that BPC 157 affects on angiogenesis by activating the NO system through $V E G F-A$ mediated $A K T$ and $p 38 / M A P K$ signaling pathways (Figure $5 \mathrm{~A}$ and $\mathrm{B}$ ).

\section{BPC I57 Attenuates Clopidogrel-Induced ER Stress and Enhances the eNOS}

\section{Activation}

Previous researches have demonstrated that endothelial NOS (eNOS) is the downstream target gene of AKT and p38/MAPK signaling pathway. Upon activation of these two pathways, eNOS leads to the production of NO that translates to an enhanced vasodilation, vascular remodeling and angiogenesis. ${ }^{41,42}$ In addition, Wang et $\mathrm{al}^{43}$ confirmed that ER stress decreases the eNOS phosphorylation and the generation of NO in rats' aortic endothelial cells, which can be reversed by ER stress inhibitors. As our previous study confirmed that Clopidogrel activates the p38/MAPK signaling pathway followed by up-regulation of C/EBP homologous transcription factor $(\mathrm{CHOP})$, and culminating with ER stress apoptosis in human gastric mucosal epithelial cell line GES- $1 .{ }^{10}$ Therefore, in the current work, we focused on detecting the expression of CHOP and eNOS proteins. As shown in Figure 5C, Clopidogrel increased the ER stress marker $C H O P$ expression and inhibited the eNOS phosphorylation compared with the control group, and these changes were both reversed by BPC 157 treatment. The addition of L-NAME partly abolished the effect of BPC 157 . These indicated that Clopidogrel induced ER stress not only promotes the gastric mucosal cells apoptosis but also reduce the release of $e N O S$-mediated NO products. And BPC 157 can inhibit ER stress and increase eNOSmediated NO product release. The effects of ER stress inhibition and eNOS increased induced by BPC 157 can be partially abolished when using the NO blocker L-NAME.

\section{Discussion}

Clopidogrel is one of the most widely used medicines world-wide due to its antiplatelet property. However, recent studies have revealed that Clopidogrel can cause various side effects in the gastrointestinal tract, including gastrointestinal ulcers, bleeding and perforation, and these complications may be fatal. ${ }^{4}$ Thus, chronic Clopidogrel users are often provided with prophylactic therapy aimed at neutralizing gastric acidity such as PPI to prevent gastric mucosal damage. But, prolonged PPI use can cause set of long-term side effects like osteoporosis-related fractures and reduce the antiplatelet effect of Clopidogrel for 


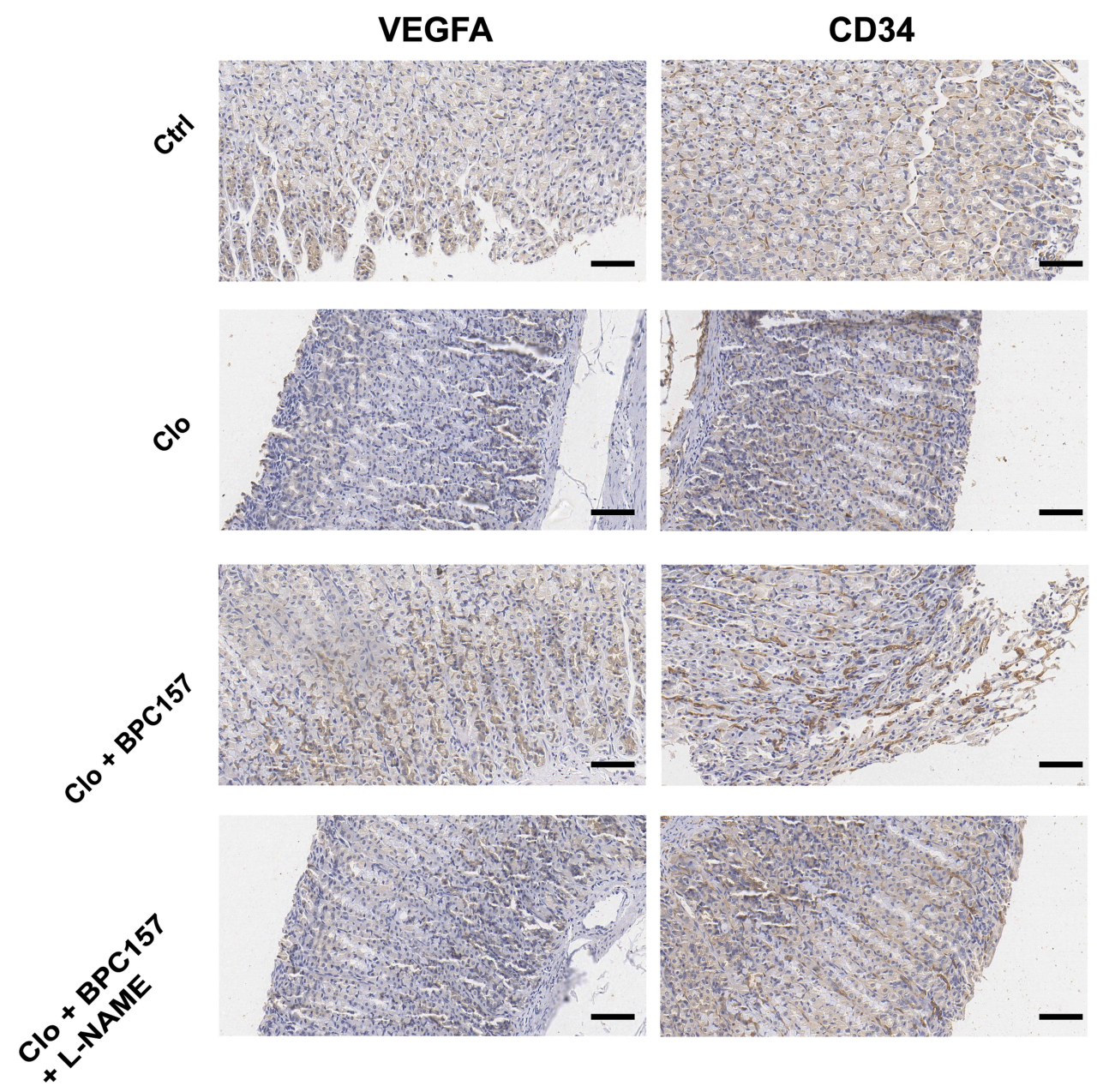

Figure 4 Immunohistochemical detection for the angiogenesis markers VEGF-A and CD34. IHC staining for VEGF-A and CD34 in rats' gastric mucosal tissue. The levels of VEGF-A and CD34 were highly expressed in Control group. In Clopidogrel group, the levels of VEGF-A and CD34 were both decreased compared with control group. And in the BPC 157 co-treatment group, the levels of these two angiogenesis markers were all up-regulated compared with Clopidogrel group. But the NO synthase blocker L-NAME inhibited up-regulation of angiogenesis markers VEGF-A and CD34 induced by BPC I57. Dark brown represents positive result. Scale bar $=50 \mu \mathrm{m}$.

preventing cardiovascular events recurrence. ${ }^{44}$ Here, we explored the utility of a natural product, BPC 157, in Clopidogrel-induced gastric injury.

Pentadecapeptide BPC 157 is a pentadecapeptide containing partial sequence of the body protection compound, which is derived from the human gastric juice. It appears to be beneficial to most of the human organs without any side effect or toxicity. Research showed that BPC 157 had anti-ulcer and anti-inflammatory effects on various gastrointestinal lesions, and also been reported to have protective effect on pancreas, endothelium, liver injuries, heart damage and pseudo arthrosis. ${ }^{45}$ The pathogenesis underlying gastric ulcer is a very complicated process. It is well known that epithelial cell proliferation/apoptosis balance plays an essential role in the multiple progressions of gastric diseases. ${ }^{46}$ In our previous study, we found Clopidogrel can cause gastric mucosal epithelial cells apoptosis then further destroy the gastric mucosal epithelial barrier. ${ }^{9}$ In accordance with these, in this study, we first observed that BPC 157 can significantly reduce the Clopidogrel-induced gastric ulcer recurrence rate in rats, and inhibit gastric mucosal epithelial cells apoptosis and inflammation. In addition, the NO blockade by L-NAME could partially reverse the protective effect of BPC 157 on gastric mucosa, so we believe that the NO system is related to the mechanism of how BPC 157 protect Clopidogrel-induced gastric mucosal injury. Since Sikiric et $\mathrm{al}^{47}$ firstly demonstrated that NO generation in gastric mucosa contributes to the anti-ulcer effect of BPC 157 in gastric lesion, subsequently, several reports analyzed the role of NO modulation in the healing effect of BPC 157 in different tissue injuries by using NO-synthase-blockade L-NAME or NO-system overstimulation L-arginine. For the treatment of colitis, ischemia and reperfusion in rats, 

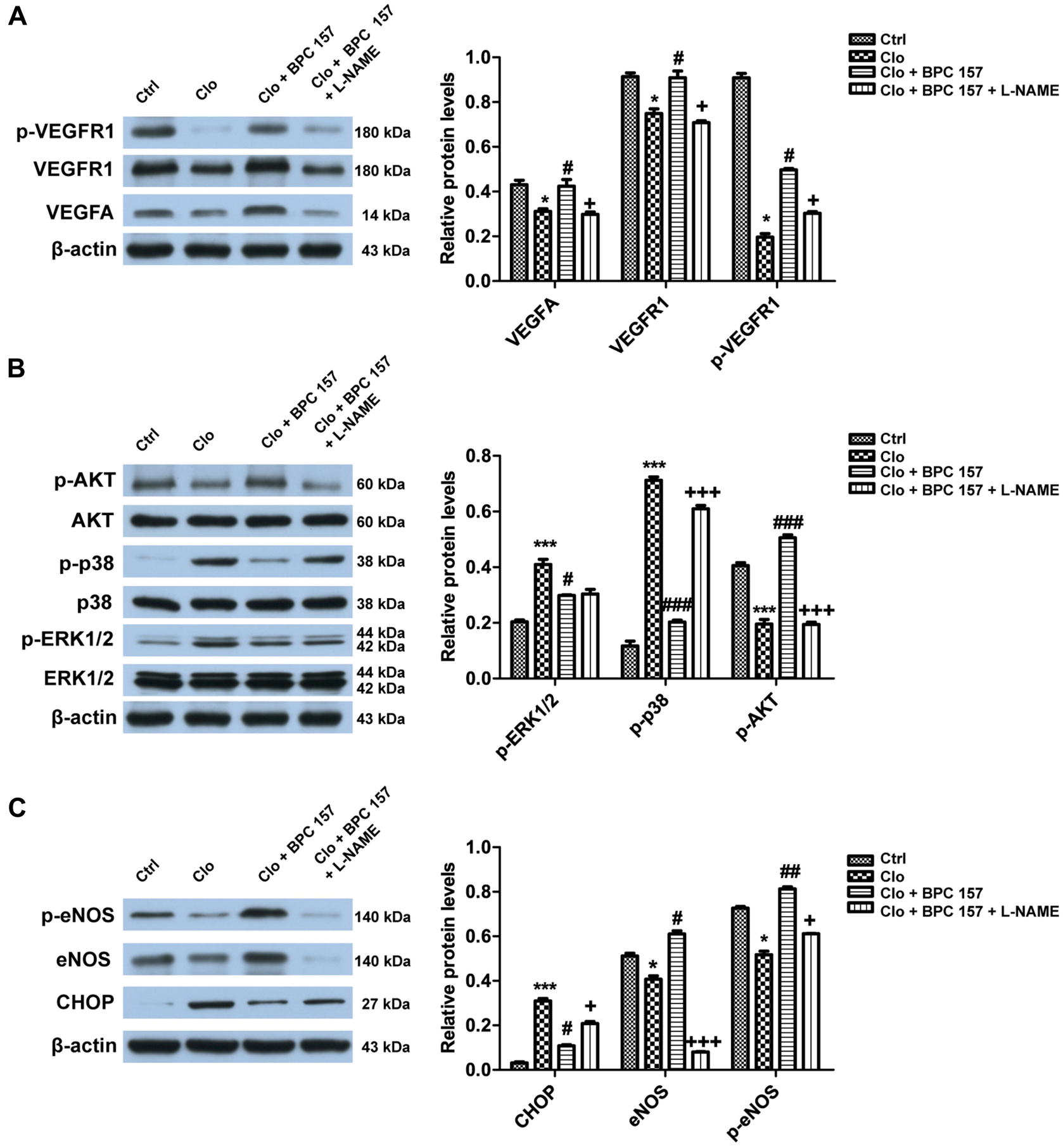

Figure 5 Role of VEGF-A-mediated-AKT or p38/MAPK signaling pathway in the inhibition of BPC I57 on Clopidogrel-induced gastric mucosal injury. (A) Clopidogrel group down-regulated the VEGF-A and VEGFRI compared with Clopidogrel group. But after administration of BPC I57, the VEGF-A and VEGFRI were up-regulated. However, the L-NAME reversed this effect of BPC I57. ${ }^{* P}<0.05$ vs Control; ${ }^{\#} P<0.05$ vs Clopidogrel; ${ }^{+} P<0.05$ vs Clopidogrel + BPC I57. (B) As the VEGF-A was downregulated, Clopidogrel dephosphorylated the AKT signaling pathway, phosphorylated $p 38 / M A P K$ and ERKIMAPK signaling pathways; but the effect of BPC I57 on these three signal pathways was completely opposite to that of Clopidogrel. Notably, L-NAME reversed the effect of AKT and p38/MAPK induced by BPC I57, but not ERKIMAPK. **** < 0.00 I

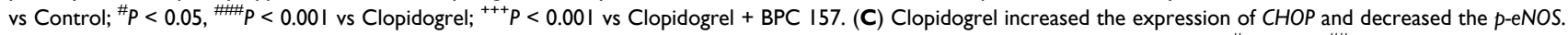
These were reversed by BPC I 57 treatment. But L-NAME partly abolished the effect of BPC I $57 .{ }^{*} P<0.05$, ${ }^{* * * P}<0.00$ I vs Control; ${ }^{\#} P<0.05$, ${ }^{\#} P<0.0$ I vs Clopidogrel; ${ }^{+} P<0.05,{ }^{+++} P<0.001$ vs Clopidogrel + BPC 157.

BPC 157 quickly restores blood supply to the chemically injured area and counteracts worsening effects induced by L-NAME or L-arginine. ${ }^{48}$ Drmic et $\mathrm{al}^{25}$ found that high- dose celecoxib administration, as a result of NO system dysfunction, induced gastrointestinal, liver and brain lesions in rats can be attenuated by BPC 157 or 
L-arginine. Similarly, BPC 157 also works against cyclophosphamide-induced hemorrhagic cystitis with severe disturbance of the NO system aggravated by using L-NAME. $^{49}$

Angiogenesis plays a key role in the healing of gastric ulcers, because the neovasculature provides blood and nutrients to the healing tissues. Konturek et $\mathrm{al}^{50}$ showed that endogenous NO can not only increase blood flow at the edge of the ulcer but also stimulate capillary angiogenesis, so it plays an important role in the healing of gastric ulcers. Therefore, in this study, we next detected angiogenesis markers, $V E G F-A$ and $C D 34$. Our immunohistochemical results showed that BPC 157 did promote the $V E G F-A$ and $C D 34$ protein synthesis, and this effect can be suppressed by L-NAME. We concluded that BPC 157 initiated angiogenesis and then further repaired the gastric mucosa through NO response. This is consistent with Domagoj Drmic's ${ }^{25}$ study which showed that Celecoxibinduced gastrointestinal mucosa injury in rats could be counteracted by BPC 157, but aggravated by L-NAME; and Zeljko Djakovic's ${ }^{51}$ study which demonstrated that the fistula formed after esophagogastric anastomosis in rats could be cured by BPC 157 and aggravated by L-NAME.

Angiogenesis is regulated by angiogenic growth factors, such as vascular endothelial growth factor (VEGF)-A. ${ }^{52} V E G F-A$ is reported to play a crucial role in the healing of gastric ulcer. Injection of a plasmid overexpressing VEGF-A in rats can promote the healing of ulcers, while the anti- $V E G F-A$ antibody antagonizes this effect. $^{53}$ The biological function of $V E G F-A$ depends on its specific receptors, such as VEGFR1, VEGFR2 and VEGFR3. VEGFR1 and VEGFR2 are endothelial cell specific, whereas $V E G F R 3$ has non-endothelial-cell functions. $V E G F-A$ binds to $V E G F R 1$ with a 10 -fold higher affinity than VEGFR2, and in Takehito Sato's ${ }^{54}$ study it was shown that VEGFR1 signaling pathway is involved in the healing of NSAID induced gastric ulcer. We hypothesized that VEGF-A-VEGFR1 may influence the healing of the ulcer induced by Clopidogrel. So in this study, we examined the relative contributions of $V E G F-A$ and $V E G F R 1$ to gastric ulcer healing in rats. The results elucidated that Clopidogrel induced recurrence of gastric ulcers and inhibited the expression of $V E G F-A$ and $V E G F R 1$ while BPC 157 promoted the healing of ulcers and upregulated both of these proteins, and the L-NAME delayed the gastric ulcer healing through downregulating the $V E G F-A$ and VEGFR1.
VEGFR1 was confirmed to autophosphorylate at Tyr$1169,1213,1242,1327$ and 1333 , and $P I-3 K$ is a potential candidate mediating biological effects after Tyr-1213 autophosphorylation. ${ }^{55}$ Several distinct signaling pathways such as $A K T, p 38 / M A P K$ and $E R K / M A P K$ are activated in a $P I-3 K$-dependent manner. This study found that BPC 157 could reverse Clopidogrel-induced $A K T$ dephosphorylation, p38/MAPK and ERK/MAPK phosphorylation. Moreover, inhibition of NO activity by L-NAME attenuated the effects of BPC 157 on $A K T$ and $p 38 / M A P K$ but not the ERK/ $M A P K$ signaling pathway, indicating that BPC 157 promotes angiogenesis and repairs gastric mucosa by activating NO activity and are related to $A K T$ and $p 38 / M A P K$ signaling pathways. The $A K T$ and $p 38 / M A P K$ pathways are both critical for cellular growth and survival. ${ }^{56,57}$ Besides these, it is well known that the $A K T$ and $p 38 / M A P K$ signaling pathways are involved in angiogenesis, and these pathways participate in multiple stages of this process. Our data are consistent with recent data generated in human umbilical vein endothelial cells such as inhibition of $A K T$ and $\mathrm{p3} 8$ / $M A P K$ pathways has antiangiogenic effects. ${ }^{58} \mathrm{Up}$ regulation of $A K T$ and $M A P K$ signaling pathways were reported to promote angiogenesis and enhance wound healing in the male diabetic mice skin wound models. ${ }^{59}$

Considering that phosphorylation of $A K T$ activates endothelial NOS (eNOS) subsequently, then leads to NO production, which can simulate vasodilation, vascular remodeling and angiogenesis; ${ }^{60}$ also increased phosphorylation of $p 38 / M A P K$ and $E R K / M A P K$ contributes to the decreased $e N O S$ activity, ${ }^{42}$ we hypothesized that $e N O S$ derived NO production may influence the healing of the gastric ulcer. Therefore, in the present study, we examined the relative contributions of $e N O S$ to gastric ulcer healing after BPC 157 treatment in rats and, as expected, the $A K T$ phosphorylation, $p 38 / M A P K$ dephosphorylation and the phosphorylated eNOS expression was increased significantly compared to Clopidogrel group. The L-NAME obviously inhibited the eNOS expression.

In our previous study, $p 38 / M A P K$ was phosphorylated by Clopidogrel in human gastric mucosal epithelial cell line GES-1, and the cell proliferation was inhibited by ER stress related transcription factor $C H O P$ activation. ${ }^{10} \mathrm{ER}$ stress is proven to induce endothelial dysfunction by disrupting vasoactive homeostasis of the endothelium. Loinard et $\mathrm{al}^{61}$ have confirmed that phosphorylated eNOS is upregulated in $\mathrm{CHOP}_{-}-\mathrm{-}$ mice and $\mathrm{CHOP}$ binds to eNOS promotor, subsequently inhibiting NO production in endothelial cells. Recent study has shown that the 
novel P2Y12 receptor antagonist, Ticagrelor upregulated the expression of phosphorylated eNOS and alleviated ER stress in the rat aortic endothelial cells, however this effect can be reversed by ER stress inhibitors. ${ }^{37}$ In our study, we found that Clopidogrel downregulated the expression of phosphorylated eNOS and induced ER stress in the rat gastric mucosa cells and the BPC 157 protected against Clopidogrel-induced gastric mucosa dysfunction by alleviating ER stress and promoting eNOS-mediated angiogenesis whereas the effect of L-NAME is completely the opposite. It is interesting that, same as P2Y12 blockers, Ticagrelor and Clopidogrel have the opposite role in ER stress and eNOS phosphorylation. We speculated whether this difference may be due to the different types of cells. The network of ER stress and the NO system will be further improved in our future research.

\section{Conclusion}

In summary, our study demonstrated that BPC 157 was able to inhibit ER stress-induced apoptosis and inflammation of gastric mucosal cells, stimulate gastric angiogenesis and then alleviate the Clopidogrel-induced gastric mucosal damage. The specific mechanism may be by activating $V E G F-A / V E G F R 1$-mediated $A K T$ and $p 38 /$ $M A P K$ signaling pathways to upregulate downstream eNOS expression, which may increase the NO product synthesis thus promote angiogenesis of gastric mucosa, and also interact with ER stress. We hope that our results in this present study could provide further evidence for the anti-ulcer effects of BPC 157 and its underlying molecular mechanisms, and also a theoretical basis for the prevention and treatment of gastric damage caused by Clopidogrel. In future, we plan to use the RNAi (RNA interference) method both at the cellular and animal levels to further investigate in-depth about the mechanism, especially the network between ER stress and the NO system.

\section{Acknowledgments}

This work was funded by grants from the National Natural Science Foundation of China (NO.81600415\&8160 0429\&81570503), Postgraduate Research and Practice Innovation Program of Jiangsu Province (No. KYCX18_0178), Southeast University's basic scientific research support (NO.3290004407) and the Nanjing Municipal Science and Technology Bureau (NO. 201715012). All of the above mentioned foundations had no role in study design, data collection and analysis, decision to publish, or preparation of the manuscript.

\section{Disclosure}

The authors report no conflicts of interest in this work.

\section{References}

1. Xie HG, Zou JJ, Hu ZY, et al. Individual variability in the disposition of and response to Clopidogrel: pharmacogenomics and beyond. Pharmacol Ther. 2011;129:267-289. doi:10.1016/j.pharmthera.2010.10.001

2. Huang KW, Luo JC, Leu HB, et al. Risk factors for upper gastrointestinal bleeding in coronary artery disease patients receiving both aspirin and Clopidogrel. J Chin Med Assoc. 2013;76:9-14. doi:10.1016/j.jcma.2012.09.004

3. Fork FT, Lafolie P, Toth E, et al. Gastroduodenal tolerance of $75 \mathrm{mg}$ Clopidogrel versus $325 \mathrm{mg}$ aspirin in healthy volunteers: a gastroscopic study. Scand J Gastroenterol. 2000;35:464-469. doi:10.1080/003655200750023705

4. Chan FK, Ching JY, Hung LC, et al. Clopidogrel versus aspirin and esomeprazole to prevent recurrent ulcer bleeding. $N$ Engl $J$ Med. 2005;352:238-244. doi:10.1056/NEJMoa042087

5. Lai KC, Chu KM, Hui WM, et al. Esomeprazole with aspirin versus Clopidogrel for prevention of recurrent gastrointestinal ulcer complications. Clin Gastroenterol Hepatol. 2006;4:860-865. doi:10.1016/j.cgh.2006.04.019

6. Hsu PI, Lai KH, Liu CP. Esomeprazole with Clopidogrel reduces peptic ulcer recurrence, compared with Clopidogrel alone, in patients with atherosclerosis. Gastroenterology. 2011;140:791-798. doi:10.1053/j. gastro.2010.11.056

7. Sibilia V, Pagani F, Lattuada N, et al. Ticlopidine prevents the formation but delays the healing of ethanol-induced gastric lesions in the rat. Pharmacol Res. 2007;55:418-425. doi:10.1016/j.phrs.2007. 01.017

8. Luo JC, Peng YL, Chen TS, et al. Clopidogrel inhibits angiogenesis of gastric ulcer healing via downregulation of vascular endothelial growth factor receptor 2. J Formos Med Assoc. 2016;115:764-772.

9. Wu HL, Gao X, Jiang ZD, et al. Attenuated expression of the tight junction proteins is involved in Clopidogrel-induced gastric injury through p38 MAPK activation. Toxicology. 2013;304:41-48. doi:10.1016/j.tox.2012.11.020

10. Wu HL, Duan ZT, Jiang ZD, et al. Increased endoplasmic reticulum stress response is involved in Clopidogrel-induced apoptosis of gastric epithelial cells. PLoS One. 2013;8:e74381. doi:10.1371/journal. pone. 0074381

11. Wu HL, Ming W, Tan JC, et al. Role of MKP-5-p38/MAPK pathway in Clopidogrel-induced gastric mucosal epithelial cells apoptosis and tight junction dysfunction. Am J Transl Res. 2020;12:1741.

12. Luo JC, Shin VY, Liu ES, et al. Non-ulcerogenic dose of dexamethasone delays gastric ulcer healing in rats. $J$ Pharmacol Exp Ther. 2003;307:692-698. doi:10.1124/jpet.103.055202

13. Luo JC, Shin VY, Liu ES, et al. Dexamethasone delays ulcer healing by inhibition of angiogenesis in rat stomachs. Eur J Pharmacol. 2004;485:275-281. doi:10.1016/j.ejphar.2003.11.038

14. Amic F, Drmic D, Bilic Z, et al. Bypassing major venous occlusion and duodenal lesions in rats, and therapy with the stable gastric pentadecapeptide BPC 157, L-NAME and L-arginine. World J Gastroenterol. 2018;24:5366-5378. doi:10.3748/wjg.v24. i47.5366

15. Kolovrat M, Gojkovic S, Krezic I, et al. Pentadecapeptide BPC 157 resolves Pringle maneuver in rats, both ischemia and reperfusion. World J Hepatol. 2020;12:184-206. doi:10.4254/wjh. v12.i5.184

16. Vukojevic J, Vrdoljak B, Malekinusic D, et al. The effect of pentadecapeptide BPC 157 on hippocampal ischemia/reperfusion injuries in rats. Brain Behav. 2020;10:e01726. doi:10.1002/ brb3.1726 
17. Hsieh MJ, Liu HT, Wang CN, et al. Therapeutic potential of pro-angiogenic BPC 157 is associated with VEGFR2 activation and up-regulation. J Mol Med. 2017;95:323-333.

18. Huang T, Zhang K, Sun L, et al. Body protective compound-157 enhances alkali-burn wound healing in vivo and promotes proliferation, migration, and angiogenesis in vitro. Drug Des Devel Ther. 2015;9:2485. doi:10.2147/DDDT.S82030

19. Chang C-H, Tsai W-C, Lin M-S, et al. The promoting effect of pentadecapeptide BPC 157 on tendon healing involves tendon outgrowth, cell survival, and cell migration. $J$ Appl Physiol. 2011;110:774-780. doi:10.1152/japplphysiol.00945.2010

20. Sikiric P, Seiwerth S, Brcic L, et al. Revised Robert's cytoprotection and adaptive cytoprotection and stable gastric pentadecapeptide BPC 157. Possible significance and implications for novel mediator. Curr Pharm Des. 2010;16:1224-1234. doi:10.2174/138161210790945977

21. Sikiric P, Seiwerth S, Rucman R, et al. Stable gastric pentadecapeptide BPC 157: novel therapy in gastrointestinal tract. Curr Pharm Des. 2011;17:1612-1632. doi:10.2174/138161211796196954

22. Ilic S, Drmic D, Zarkovic K, et al. Ibuprofen hepatic encephalopathy, hepatomegaly, gastric lesion and gastric pentadecapeptide BPC 157 in rats. Eur $J$ Pharmacol. 2011;667:322-329. doi:10.1016/j. ejphar.2011.05.038

23. Lojo N, Rasic Z, Zenko Sever A, et al. Effects of diclofenac, L-NAME, L-arginine, and pentadecapeptide BPC 157 on gastrointestinal, liver, and brain lesions, failed anastomosis, and intestinal adaptation deterioration in 24 hour-short-bowel rats. PLoS One. 2016;11:e0162590. doi:10.1371/journal.pone.0162590

24. Sikiric P, Seiwerth S, Rucman R, et al. Toxicity by NSAIDs. Counteraction by stable gastric pentadecapeptide BPC 157. Curr Pharm Des. 2013;19:76-83.

25. Drmic D, Kolenc D, Ilic S, et al. Celecoxib-induced gastrointestinal, liver and brain lesions in rats, counteraction by BPC 157 or L-arginine, aggravation by L-NAME. World $J$ Gastroenterol. 2017;23:5304-5312. doi:10.3748/wjg.v23.i29.5304

26. Becejac T, Cesarec V, Drmic D, et al. An endogeous defensive concept, renewed cytoprotection/adaptive cytoprotection: intra(per)-oral/intragastric strong alcohol in rat. Involvement of pentadecapeptide BPC 157 and nitric oxide system. $J$ Physiol Pharmacol. 2018;69.

27. Stupnisek M, Franjic S, Drmic D, et al. Pentadecapeptide BPC 157 reduces bleeding time and thrombocytopenia after amputation in rats treated with heparin, warfarin or aspirin. Thromb Res. 2012;129:652-659. doi:10.1016/j.thromres.2011.07.035

28. Stupnisek M, Kokot A, Drmic D, et al. Pentadecapeptide BPC 157 reduces bleeding and thrombocytopenia after amputation in rats treated with heparin, Warfarin, L-NAME and L-arginine. PLoS One. 2015;10:e0123454. doi:10.1371/journal.pone.0123454

29. Sikiric P, Seiwerth S, Grabarevic Z, et al. The beneficial effect of BPC 157, a 15 amino acid peptide BPC fragment, on gastric and duodenal lesions induced by restraint stress, cysteamine and $96 \%$ ethanol in rats. A comparative study with $\mathrm{H} 2$ receptor antagonists, dopamine promotors and gut peptides. Life Sci. 1994;54:PL63-68. doi:10.1016/0024-3205(94)00796-9

30. Sikiric P, Separovic J, Anic T, et al. The effect of pentadecapeptide BPC 157, H2-blockers, omeprazole and sucralfate on new vessels and new granulation tissue formation. $J$ Physiol Paris. 1999;93:479-485. doi:10.1016/S0928-4257(99)00123-0

31. Okabe S, Roth JLA, Pfeiffer CJ. Roth JL and Pfeiffer CJ. A method for experimental, penetrating gastric and duodenal ulcers in rats. Observations on normal healing. Am J Dig Dis. 1971;16:277-284. doi:10.1007/BF02235252

32. Antonisamy P, Duraipandiyan V, Aravinthan A, et al. Protective effects of friedelin isolated from Azima tetracantha Lam. against ethanol-induced gastric ulcer in rats and possible underlying mechanisms. Eur J Pharmacol. 2015;750:167-175. doi:10.1016/j. ejphar.2015.01.015
33. Jahovic N, Erkanli G, Iseri $\mathrm{S}$, et al. Gastric protection by alpha-melanocyte-stimulating hormone against ethanol in rats: involvement of somatostatin. Life Sci. 2007;80:1040-1045. doi:10.1016/j. 1fs.2006.11.036

34. Ng F, Wong S, Chang C, et al. High incidence of Clopidogrelassociated gastrointestinal bleeding in patients with previous peptic ulcer disease. Aliment Pharmacol Ther. 2003;18:443-449. doi:10.1046/j.1365-2036.2003.01693.x

35. Sakaki N, Takemoto TJ. The relationship between endoscopic findings of gastric ulcer scar and ulcer relapse. J Clin Gastroenterol. 1993;17:S64-69. doi:10.1097/00004836-199312001-00014

36. Saran U, Mani KP, Balaguru UM, et al. sFRP4 signalling of apoptosis and angiostasis uses nitric oxide-cGMP-permeability axis of endothelium. Nitric Oxide. 2017;66:30-42. doi:10.1016/j. niox.2017.02.012

37. Buijs N, Oosterink JE, Jessup M, et al. A new key player in VEGF-dependent angiogenesis in human hepatocellular carcinoma: dimethylarginine dimethylaminohydrolase 1. Angiogenesis. 2017;20:557-565. doi:10.1007/s10456-017-9567-4

38. Tjaden K, Adam C, Godfrey R, et al. Low density lipoprotein interferes with intracellular signaling of monocytes resulting in impaired chemotaxis and enhanced chemokinesis. Int $J$ Cardiol. 2018;255:160-165. doi:10.1016/j.ijcard.2017.11.109

39. Barthomeuf $C$, Lamy S, Blanchette $M$, et al. Inhibition of sphingosine-1-phosphate- and vascular endothelial growth factor-induced endothelial cell chemotaxis by red grape skin polyphenols correlates with a decrease in early platelet-activating factor synthesis. Free Radic Biol Med. 2006;40:581-590. doi:10.1016/j. freeradbiomed.2005.09.015

40. $\mathrm{Xu} \mathrm{T}, \mathrm{Lv} \mathrm{Z}$, Chen Q, et al. Vascular endothelial growth factor over-expressed mesenchymal stem cells-conditioned media ameliorate palmitate-induced diabetic endothelial dysfunction through PI-3K/AKT/m-TOR/eNOS and p38/MAPK signaling pathway. Biomed Pharmacother. 2018;106:491-498. doi:10.1016/j. biopha.2018.06.129

41. Wang X, Zhan E, Lu G, et al. Sphingosine-1-phosphate improves the biological features of mouse bone marrow-derived EPCs partially through PI3K/AKT/eNOS/NO pathway. Molecules. 2019;24:2404. doi:10.3390/molecules24132404

42. Jia Y, Guo B, Yang W, et al. Rho kinase mediates Porphyromonas gingivalis outer membrane vesicle-induced suppression of endothelial nitric oxide synthase through ERK1/2 and p38 MAPK. Arch Oral Biol. 2015;60:488-495. doi:10.1016/j.archoralbio.2014.12.009

43. Wang X, Han X, Li M, et al. Ticagrelor protects against AngII-induced endothelial dysfunction by alleviating endoplasmic reticulum stress. Microvasc Res. 2018;119:98-104. doi:10.1016/j. mvr.2018.05.006

44. Johnson DA, Oldfield EC. Reported side effects and complications of long-term proton pump inhibitor use: dissecting the evidence. Clin Gastroenterol Hepatol. 2013;11:458-464. doi:10.1016/j. cgh.2012.11.031

45. Sikiric P. The pharmacological properties of the novel peptide BPC 157. Inflammopharmacology. 1999;7:1-14. doi:10.1007/s10787-9990022-z

46. Tamura M, Matsui H, Kaneko T, et al. Alcohol is an oxidative stressor for gastric epithelial cells: detection of superoxide in living cells. J Clin Biochem Nutr. 2013;53:75-80. doi:10.3164/jcbn.13-32

47. Sikiric P, Seiwerth S, Grabarevic Z, et al. The influence of a novel pentadecapeptide, BPC 157, on N(G)-nitro-L-arginine methylester and L-arginine effects on stomach mucosa integrity and blood pressure. Eur J Pharmacol. 1997;332:23-33. doi:10.1016/S00142999(97)01033-9

48. Duzel A, Vlainic J, Antunovic M, et al. Stable gastric pentadecapeptide BPC 157 in the treatment of colitis and ischemia and reperfusion in rats: new insights. World J Gastroenterol. 2017;23:8465-8488. doi:10.3748/wjg.v23.i48.8465 
49. Sucic M, Luetic K, Jandric I, et al. Therapy of the rat hemorrhagic cystitis induced by cyclophosphamide. Stable gastric pentadecapeptide BPC 157, L-arginine, L-NAME. Eur $J$ Pharmacol. 2019;861:172593. doi:10.1016/j.ejphar.2019.172593

50. Konturek SJ, Brzozowski T, Majka J, et al. Inhibition of nitric oxide synthase delays healing of chronic gastric ulcers. Eur J Pharmacol. 1993;239:215-217. doi:10.1016/0014-2999(93)90997-V

51. Djakovic Z, Djakovic I, Cesarec V, et al. Esophagogastric anastomosis in rats: improved healing by BPC 157 and L-arginine, aggravated by L-NAME. World $J$ Gastroenterol. 2016;22:9127-9140. doi:10.3748/wjg.v22.i41.9127

52. Shibuya M. Vascular endothelial growth factor receptor-1 (VEGFR-1/Flt-1): a dual regulator for angiogenesis. Angiogenesis. 2006;9:225-230; discussion 231. doi:10.1007/s10456-006-9055-8

53. Jones MK, Kawanaka H, Baatar D, et al. Gene therapy for gastric ulcers with single local injection of naked DNA encoding VEGF and angiopoietin-1. Gastroenterology. 2001;121:1040-1047. doi:10.1053/ gast.2001.29308

54. Sato T, Amano H, Ito $\mathrm{Y}$, et al. Vascular endothelial growth factor receptor 1 signaling facilitates gastric ulcer healing and angiogenesis through the upregulation of epidermal growth factor expression on VEGFR1+ CXCR4+ cells recruited from bone marrow. J Gastroenterol. 2014;49:455-469. doi:10.1007/s00535-013-0869-z

55. Shibuya M. Differential roles of vascular endothelial growth factor receptor-1 and receptor-2 in angiogenesis. $J$ Biochem Mol Biol. 2006;39:469-478.
56. Franke TF, Kaplan DR, Cantley LC. PI3K: downstream AKTion blocks apoptosis. Cell. 1997;88:435-437. doi:10.1016/S00928674(00)81883-8

57. Ki YW, Park JH, Lee JE, et al. JNK and p38 MAPK regulate oxidative stress and the inflammatory response in chlorpyrifos-induced apoptosis. Toxicol Lett. 2013;218:235-245. doi:10.1016/j.toxlet.2013.02.003

58. Kim GD. Kaempferol inhibits angiogenesis by suppressing HIF-1 $\alpha$ and VEGFR2 activation via ERK/p38 MAPK and PI3K/Akt/mTOR signaling pathways in endothelial cells. Prev Nutr Food Sci. 2017;22:320. doi:10.3746/pnf.2017.22.4.320

59. Yuan X, Han L, Fu P, et al. Cinnamaldehyde accelerates wound healing by promoting angiogenesis via up-regulation of PI3K and MAPK signaling pathways. Lab Invest. 2018;98:783-798. doi:10.1038/s41374-018-0025-8

60. Dimmeler S, Fleming I, Fisslthaler B, et al. Activation of nitric oxide synthase in endothelial cells by Akt-dependent phosphorylation. Nature. 1999;399:601-605. doi:10.1038/21224

61. Loinard C, Zouggari Y, Rueda P, et al. C/EBP homologous protein-10 (CHOP-10) limits postnatal neovascularization through control of endothelial nitric oxide synthase gene expression. Circulation. 2012;125:1014-1026. doi:10.1161/CIRCULATIONAHA.111.04 1830

\section{Publish your work in this journal}

Drug Design, Development and Therapy is an international, peerreviewed open-access journal that spans the spectrum of drug design and development through to clinical applications. Clinical outcomes, patient safety, and programs for the development and effective, safe, and sustained use of medicines are a feature of the journal, which has also been accepted for indexing on PubMed Central. The manuscript management system is completely online and includes a very quick and fair peer-review system, which is all easy to use. Visit http://www. dovepress.com/testimonials.php to read real quotes from published authors. 